\title{
Whispering gallery modes of silica nanospheres for absorption enhancement in ultra-thin c-Si solar cells
}

\author{
P. Banerjee ${ }^{1}$, A. B. Roy ${ }^{1}$, A. Dhar ${ }^{1}$, M. Choudhuri ${ }^{2}$, S. Das ${ }^{1}$ and A. Kundu ${ }^{1 *}$ \\ ${ }^{1}$ Centre of Excellence for Green Energy and Sensor Systems (CEGESS), Indian Institute of Engineering Science \\ and Technology (IIEST), Shibpur, 711103 India \\ ${ }^{2}$ Department of ECE, Dr.Sudhir Chandra Sur Degree Engineering College, Dumdum, 700074, India \\ *Email: kunduavra@gmail.com
}

Enhancing the photon absorption is the most significant challenge for ultra-thin crystalline silicon solar cells. One approach is to put an Anti-Reflection Coating (ARC) to reduce the reflection $(\sim 2 \%)$ and subsequently increase the absorption as a bare silicon surface reflects $\sim 30 \%$ of the incident photon energy. But reducing reflection does not ensure enhanced absorption in case of ultra-thin cells, as advanced light management techniques are required to do the same. Here we present an ingenious approach of light trapping by whispering gallery modes of silica nanospheres. These dielectric nanospheres synthesized by Stober method act as miniature domes where the light travels through the internal walls of the nanospheres causing absorption enhancement in the nanosphere/ silicon interface[2]. A mono-layer of silica nanosphere is formed on silica substrate by dipcoating. Simulation of silica nanospheres on an active silicon substrate $(3 \mu \mathrm{m})$ have been done using Rigorous Coupled Wave Analysis (RCWA) method. Further, the nanospherical geometry ensures a wide angle light collection and low reflection losses. Hence, this structure can be an answer to both broadband light absorption and omnidirectional light trapping problem. A $25 \%$ increment in absorption is observed in simulation for structure with silica nanospheres compared to bare silicon structure. Figure 1 shows the simulation model done using RCWA method and Figure 2 shows the SEM image of synthesized silica nanospheres. The plot from experimental data in Figure 2 inset clearly shows the reduction in reflectance due to the silica nanoparticles.

\section{References}

1. J. Grandidier et al, "Gallium Arsenide Solar Cell Absorption Enhancement Using Whispering Gallery
Modes of Dielectric Nanospheres," in IEEE Journal of Photovoltaics, vol. 2, no. 2, pp. 123-128, April 2012. doi: 10.1109/JPHOTOV.2011.2180512

2. Sonali Das et al, "Silica nanoparticles on front glass for efficiency enhancement in superstrate-type amorphous silicon solar cells," JOURNAL OF PHYSICS D: APPLIED PHYSICS, 46 (2013) 415102(10pp).doi:10.1088/0022-727/46/41/415102

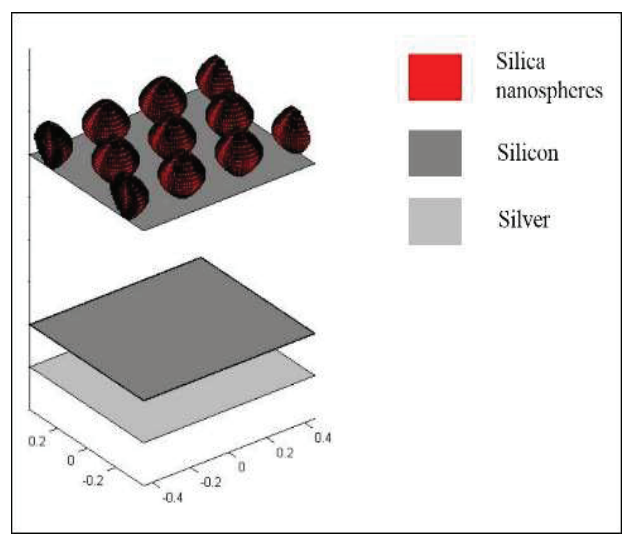

Figure 1: Structure simulated in MATLAB

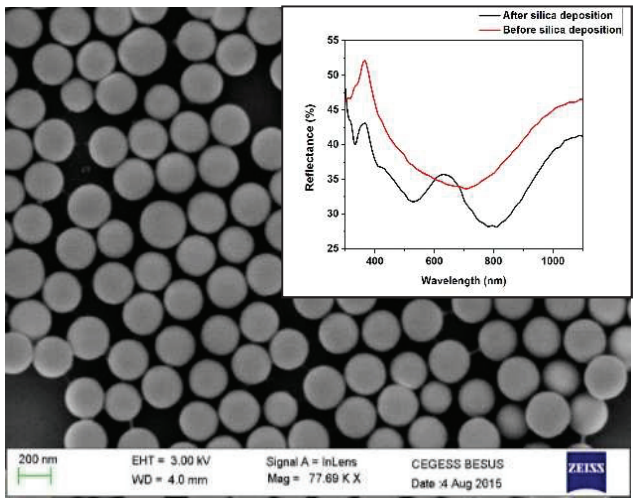

Figure 2: SEM image of silica Nanoparticles, Inset: Change in reflectance after silica deposition 